\title{
Cooperación regional ante un mundo que se integra'
}

\section{Alfredo Guerra-Borges}

En los últimos tiempos el tema de la integración económica ha cobrado inusitada relevancia. Aunque en el discurso politico la integración ya habla reaparecido en anos recientes, lo que constitula una reconsideración de la desdenosa actitud asumida en los anos setenta, lo nuevo ahora es que el planteamiento se hace desde una perspectiva radicalmente distinta a la tradicional. De tal manera súbito ha sido el cambio de posiciones que hay lugar para preguntarse sobre sus motivaciones. Piénsese por un momento que se necesitaron diez anos para que en el seno de la Asociación Latinoamericana de Integración se incrementara la preferencia arancelaria regional del $10 \%$ al $20 \%$. Ningún gobierno cedla posiciones, aunque en el discurso oficial la integración fuera en todo momento un tema recurrente.

Ahora, por el contrario; en el presente ano para ser más exactos, el lenguaje que se habla es de integración comercial a corto plazo. Colombia, México y Venezuela vienen explorando la posibilidad de suscribir un acuerdo de libre comercio. Chile y Venezuela firmaron el 11 de octubre recién pasado un memorándum de emtendimiento que establece la fecha máxima del 31 de mayo de 1994 para la liberalización de su comercio bilateral. Argentina y Brasil decidieron el 6 de julio del ano en curso el establecimiento de un mercado comin entre ambos palses, el cual deberá estar definitivamente conformado el 31 de diciembre de 1994. Unuguay y Paraguay se incorporarán al sistema de libre comercio argentino-brasileno. También Chile y México han suscrito un acuerdo marco para liberar en un plazo corto su comercio bilateral.

1. Disertación en la Academia Mexicana de Economla Polftica, noviembre 8, 1990. El autor es miembro de número de la Academia. 
En resumen, un fantasma recorre América Latina: el fantasma de la integración.

A nuestro parecer, esta sucesión de acontecimientos trascendentales ha sido posible porque las condiciones para integrarse más han subyacido desde hace tiempo en la integración lograda en décadas anteriores. La crisis de los 80 , que inicialmente desquició a todos los esquemas de integración de América Latina y el Caribe, finalmente dio un resultado contrario. Ante el agobio de la deuda externa, la agresión del proteccionismo de los paises desarrollados, el éxito muy precario de las politicas de ajuste, cuando no su completo fracaso, y otros tantos motivos de frustración que se han acumulado, revelaron nuevamente la necesidad y la pertinencia de la integración latinoamericana.

Todo parece indicar que hay además otros motivos. La formación de grandes bloques económicos de paises industriales no pasa por alto a los gobiemos latinoamericanos. El Acta de Buenos Aires para el establecimiento del Mercado Común argentino-brasileno, claramente alude en su primer considerando a "la evolución reciente de los acontecimientos internacionales, en especial la consolidación de los grandes espacios económicos, la globalización del escenario económico internacional y la importancia crucial de lograr una adecuada inserción económica internacional para nuestros paises...".

Pero el principal detonante quizás ha sido la reciente propuesta del Presidente Bush de continentalizar la integración. La llamada "Iniciativa de las Américas" causó sorpresa y desconcierto, además de cierta expectación ante la posibilidad de una redefinición de la política comercial de los Estados Unidos, pero también colocó a la América Latina en la situación de tener que fijar posición ante una eventualidad inesperada. Es indudable que se tiene clara conciencia de las disparidades de todo orden existentes con los Estados Unidos. Si a eso se agrega una larga historia de frustraciones y desacuerdos en las relaciones comerciales con los Estados Unidos, podria suponerse que las recientes iniciativas latinoamericanas de integración apuntan a mejorar las posiciones de negociación con los Estados Unidos.

Con lo anterior en mente se abordará en esta disertación el tema de la integración regional en los dos planos en que hoy dia se manifiesta: como respuesta a los desafios y oportunidades que plantea el entorno internacional, y como estrategia que responde a imperativos internos de desarrollo.

\section{El desafio del mundo Industrial}

El rasgo más caracteristico del escenario internacional es hoy dia la 
formación de grandes bloques económicos de los paises industriales, que rivalizan entre sí. La formalización de estos bloques no constituye un movimiento hacia el libre comercio internacional sino hacia la regionalización de los mercados e inaugura una nueva fase de la satelización de las economias periféricas.

El regionalismo de los países desarrollados tiene profundas motivaciones y conduce a una redistribución mundial del poder económico y político. A su vez, este realineamiento está vinculado a la revolución de la ciencia y la tecnologia modernas, en virtud de la cual se viene modificando radicalmente la estructura del comercio intemacional y de la producción.

Los procesos de regionalización que están en curso tienen la particularidad de agrupar a paises relativamente homogéneos. Se trata de paises con un alto nivel de desarrollo industrial y una agricultura muy tecnificada; que tienen el más alto nivel de ingreso en el mundo, o están en vias de alcanzarlo en el curso de esta década; y entre los cuales se encuentran los paises exportadores más importantes.

Se trata además de agrupaciones dentro de cuya relativa homogeneidad destacan los paises que actualmente están remodelando la economia internacional de acuerdo a las necesidades de su superdesarrollo. En relación a esto último digamos de paso que es evidente que los procesos de cambio que están en curso en América Latina tienen la obvia finalidad de recaudar las economias latinoamericanas al nuevo entorno intemacional y que la ideología que corresponde a las exigencias de este periodo de readecuación es el neoliberalismo.

\section{La Europa del 92}

Por lo que toca a la regionalización económica de Europa cabe distinguir dos dimensiones. Una es la llamada Europa del 92, que conforme el Acta Unica Europea suscrita en 1986 establecerá un mercado único (el mas grande del mundo) para el movimiento de las personas, las mercancias, los capitales y los servicios. La otra dimensión es la Europa exsocialista, cuya tendencia natural, en razón de la historia, de la economia y la política, será hacia una muy estrecha vinculación con la Europa del 92.

No está excluida la posibilidad de que en un futuro no muy lejano las economias de Europa Oriental y la Unión Soviética pasen a constituir de alguna manera una gigantesca agrupación de paises que se extienda desde Lisboa a Vladivostok, pero aun cuando asi no fuera ya ahora la transformación productiva de Europa Oriental, particularmente 
la de la Unión Soviética, absorberá un volumen enorme de capitales, con lo que se estrecharán aun más las ya reducidas opciones de crédito internacional para América Latina. Adicionalmente, la ampliación del intercambio comercial y tecnológico entre las dos Europas, concentrará la atención de ambas. No obstante las declaraciones oficiales que lo niegan, en los hechos los problemas de América Latina tendrán muy bajo pertil en el escenario europeo.

\section{Algunas consecuenclas de la Europa del 92}

No es posible predecir las consecuencias que tendrá para América Latina la formación del macromercado europeo, pero pueden adelantarse algunas hipotesis. ${ }^{2}$

Una posibilidad de signo positivo es que el impetu que imprimirá a las economias europeas el mercado único dinamice el comercio internacional y se abra asi para América Latina una oportunidad de participación. En teoria todo dependerá entonces de la capacidad y la posibilidad de los paises latinoamericanos para aprovechar las nuevas oportunidades comerciales, pero más adelante se verá que en los hechos esta hipótesis tiene importantes restricciones.

Una consecuencia sin duda favorable será la abolición de los controles de frontera y la eliminación de obstáculos técnicos al comercio. La importancia de lo primero radica en que los controles de frontera se utilizan hasta la fecha para aplicar las restricciones comerciales cuantitativas establecidas por cada pais, en particular las restricciones al comercio de textiles y prendas de vestir regulado por el Acuerdo Multifibras. Es posible que en la Europa del 92 las restricciones nacionales se condensen en una restricción cuantitativa de ámbito comunitario, 10 que favoreceria las exportaciones latinoamericanas.

Por su parte, la eliminación de los obstáculos técnicos al comercio permitirá la libre circulación, no solo de las mercancias fabricadas en el territorio de la Comunidad, sino también de las que hayan sido importadas por los paises miembros, cualquiera que sea el punto de ingreso al territorio aduanero comunitario. Esta medida favorecerá también las exportaciones latinoamericanas, pues las librará del costo que implica hoy dia satisfacer las normas técnicas de cada pais.

Junto a lo anterior pueden formularse algunas hipótesis pesimistas.

2. Ver, entre otras fuentes, Luciano Berrocal, "Perspectiva 1992: El Mercado Unico Europeo. Nuevo Desafio en las Relaciones Europa-América Latina?", en Pensamiento lberoamericano, 15, Madrid, 1989; y Banco Mundial, Informe del Desarrollo Mundial 1989. 
En primer lugar, el supuesto de que la Europa del 92 ampliará las oportunidades de exportación para América Latina contradice la tendencia que en anos recientes han mostrado las exportaciones latinoamericanas al mercado europeo. Mientras en 1979 la Comunidad Económica Europea fue el destino del $31 \%$ de las exportaciones latinoamericanas, en 1988 ese porcentaje habia caido al nivel de $21 \%{ }^{3}$

Lo anterior no puede extranar, pues América Latina ha encontrado crecientes dificultades de acceso al mercado europeo, en particular por las barreras de la Política Agricola de la Comunidad. Nada hace esperar por ahora que en el futuro se encontrará menos obstáculos.

Por el contrario, puede adelantarse la hipótesis de que el mercado único europeo provocará desequilibrios sociales internos y el resurgimiento del nacionalismo en las regiones que resulten desfavorablemente afectadas, lo que repercutirá en un incremento del proteccionismo en la Europa del 92.

De igual manera, es posible que tenga un impacto negativo en América Latina el nuevo reparto de los mercados internacionales entre Europa, Estados Unidos y el Japón, como lógica consecuencia del reforzamiento de la capacidad de negociación de la Europa del 92. De nuevo se podria pensar en el comercio de productos agrícolas, pues los precios altamente competitivos de las exportaciones agricolas de la Comunidad por efecto de los subsidios han tenido como consecuencia que América Latina haya perdido hasta ahora una tercera parte de sus mercados 4 .

\section{El convenlo de Ilbre comerclo Canadá-Estados Unldos}

El proceso de regionalización que despierta mayor interés en América Latina es el de los Estados Unidos y Canadá, cuyo Acuerdo de Libre Comercio se suscribió el 2 de junio de 1988. El Acuerdo, asi como las circunstancias que lo originaron, aportan interesantes lecciones para América Latina.

Contrariamente a lo que su nombre indica, no se trata de un tratado cuyo objeto sea simplemente eliminar los derechos arancelarios para el comercio entre ambos paises, pues el Acuerdo contiene disposiciones sobre muy diversos asuntos, entre otros, normas técnicas energia, in-

3. Mark B. Rosemberg, The Changing Hemispheric Trade Environment: Oportunities and Chanllenges for Central America, Latin American and Caribbean Center, Florida International University, 1990, p. 8 (mimiográfo).

4. Idem. 
dustria automovillstica, compras del sector público, inversión extranjera, servicios y otros más.

Los derechos arancelarios en su mayor parte serán eliminados por etapas a partir del quinto ano de vigencia del Acuerdo, y hasta los diez anos en el caso de los productos más sensibles. Por consiguiente, la zona de libre comercio se perfeccionará hasta 1988.

El Aaverdo de Libre Comercio no pone fin a los problemas en las relaciones de Estados Unidos-Canadá. Las áreas de conflicto son relativamente numerosas, pero la mención de unas pocas de ellas permitirá ilustrar el punto, y más adelante hacer algunas inferencias.

Una primera zona friccional es el de las inversiones extranjeras. $\mathbf{A}$ suscribir el Acuerdo Canadá aflojó sus regulaciones en esta materia, las cuales han sido tradicionalmente fuertes. Sin embargo, la parte norteamericana impugna la vigencia de substanciales restricciones canadienses a la inversión extranjera en el sector energético, to mismo que en las llamadas "industrias culturales", que incluyen la prensa, el cine, la televisión, las editoriales y otros aspectos.

En particular son interesantes dos casos en que claramente se transgrede el principio de la ventaja comparativa, no obstante ser uno de los temas de mayor relevancia en la prédica neoliberal. De acuerdo a lo que manifiesta un funcionario del Departamento de Comercio de los Estados Unidos, "el Canadá tiende a concentrar sus subsidios en áreas donde piensan que tienen alguna ventaja competitiva", ${ }^{\circ}$ y se menciona como principales beneficiarios los productos de la madera y la mineria.

El segundo caso es el del transporte maritimo. Hasta ahora tanto el Canadá como los Estados Unidos protegen su industria naviera mediante el requisito de que sus importaciones a través de puertos nacionales deben ser transportadas por barcos de la bandera del respectivo pais. La industria naviera del Canadá es más eficiente que la norteamericana y no abriga temor a que se desmantele la protección que se le ha venido dando, pero los Estados Unidos se oponen a tal desmantelamiento precisamente por ser los menos eficientes.

En cuanto al comercio, una de las varias zonas de desacuerdo es la oposición de los Estados Unidos a dejar sin efecto las restricciones que pesan sobre los textiles canadienses, y a eliminar sus cuotas de

5. Ann Hughes, Commerce Department, citada por Patrick G. Marshall "North America Trade Pact: A Good Idea?", Editorial Research Reports, Washington, Congressional Quaterly Inc., 1989, p. 686. 
importacion azucarera. Pero to que en particular afecta el comercio entre ambos palses son los subsidios. En lo que respecta a la industria ninguno de los dos palses puede eliminarlos mientras otros compettdores (lease los europeos, el Japón o los palses del sudeste asiátioo) subsidien a su propia industria. Por su parte, la producción agricola de ambos palses es ampliamente subsidiada, pero el Canadá se queja del atto nivel de los subsidios norteamericanos a la producción de trigo, cobada y avena, razón por la cual el Acuerdo autoriza a las partes canadienses a restringir sus importaciones de estos granos en tanto bs subsidios norteamericanos no sean iguales o menores que los precios de sustentación del Canadá.

\section{Impacto del Acuerdo Canadł-Estados Unldos en Amérlca Latina}

En cuanto a las consecuencias para América Latina del Acuerdo de Libre Comercio de Canadá y Estados Unidos podrían hacerse las siguientes consideraciones preliminares.

En primer lugar, hay que hacerse una idea de la dimensión del bloque económico constituido. Se trata de dos palses que tienen gran semejanza en sus patrones de consumo y sus niveles de ingreso. Tienen los dos un alto nivel de desarrollo industrial y sendas economlas agricolas de la mayor importancia en el comercio mundial. Estados Unidos y Canadá son además los dos mayores socios comerciales del mundo. Medido por sus exportaciones reclprocas en 1988, su intercambio alcanzó un total de \$US 151,223 millones, o sea, en ese ano fue superior en $65 \%$ al intercambio de América Latina y el Caribe con los Estados Unidos'.

Es indudable entonces que la unificación de los mercados de Canadá y Estados Unidos aumentará su peso en la economla internacional, que es precisamente lo que se busca ante la creciente pérdida de terreno frente a Europa y el Japón.

Si el análisis de las consecuencias se hace desde una perspectiva restringida al comercio, el punto de partida puede ser la hipótesis bastante convencional de que el impulso que el Acuerdo imprima a las economlas norteamericana y canadiense abrirá nuevas oportunidades de comercio para América Latina.

Tal hipótesis está sujeta a algunas restricciones. En lo referente a Canadá hay que decir que el mercado norteamericano constituye el destino del $75-80 \%$ de las exportaciones canadienses, y se puede

6. International Monetary Fund, Direction of Trade Statistics. Yeartook, 1989. 
reforzará esta corriente. Si las cosas se ven desde el punto de vista de la demanda tampco parece que América Latina pueda esperar un incremento sustantivo de las compras canadienses, pues los Estados Unidos son el principal proveedor del Canadá, y con mayor razón lo serán como resultado del Acuerdo de Libre Comercio.

Por otra parte, un análisis comparativo del comercio de América Latina con esos dos paises, revela que en 1988 el comecio de la región con los Estados Unidos tuvo un valor total de \$US 91,582 millones, mientras que el comercio con el Canadá tuvo un valor de \$US 4,977 millones, to que representa solamente un $5.4 \%$ del intercambio con los Estados Unidos. ${ }^{7}$ Por consiguiente, son los Estados Unidos, y no Canadá, el principal origen y destino del comercio de América Latina y el Caribe con el bloque del norte, y en consecuencia, podria concluirse que el impacto del Acuerdo será bastante moderado en lo tocante al comecio de Canadá con América Latina.

En lo que se refiere a la otra parte del Acuerdo (los Estados Unidos) cabe hacer las siguientes observaciones. En el periodo 1980-1989 el comercio de América Latina con los Estados Unidos muestra claramente dos fuertes tendencias. Una es que, en términos relativos, América Latina ha perdido importancia en el comercio exterior de los Estados Unidos ${ }^{8}$. Por consiguiente, se puede suponer que el crecimiento de la economia norteamericana por efecto del Acuerdo con Canadá, dará lugar a una expansión más que proporcional del comercio norteamericano hacia otras regiones, y menos intensa hacia América Latina.

Una segunda tendencia es hacia su creciente concentración en un número limitado de paises, principalmente Brasil y México. Con estos dos paises los Estados Unidos realizaron en 1980 el 47\% de su comercio total con América latina, pero en 1979 esa participación se elevó a $61 \%$. La tendencia a la concentración del comercio es aun mayor con México, pais al que correspondió un poco más de la tercera pate del intercambio total de los Estados Unidos con América latina en 1980, pero ya para 1989 esa participación se elevó a casi la mitad?.

7. Ibid.

8. Sistema Económico Latinoamericano, La situación Económica de los Estados Unidos y sus relaciones con América Latina y el Caribe, Caracas, (SP/CL/XVI. O/Di NN 8), 1990. La información disponible indica que entre 1980 y 1989 las exportaciones de los Estados Unidos a la América Latina cayeron del 17.3 al 13.5 por ciento de su exportación total, y que las importaciones desde América Latina se redujeron del 15 al 12 por ciento de la importación total de los Estados Unidoc.

9. SELA, op. cit., p. 40. 
Por consiguiente, sl el Acuerdo de Libre Comercio con Canadá dinamiza a la economla norteamericana, lo mas razonable es esperar que ese mayor dinamismo no se traducirá en un incremento significativo del comercio con América Latina sino, ante todo, en un reforzamiento de los vínculos económicos, y no solo comerciales, con México.

Por otra parte, si las importaciones latinoamericanas procedentes de los Estados Unidos siguieran incrementándose más aceleradamente que sus exportacions a este pais, como fue la tendencia en la década pasada, es de esperar que el crecimiento de la economía norteamericana se traduzca en una expansión de sus ventas a la región, lo que no aliviarla la penuria de reservas internacionales de América Latina, sino todo lo contrario.

\section{Más allá de lo comerclal}

Lo anterior constituye una aproximación preliminar limitada al intercambio comercial, pero el problema debe ubicarse dentro de la perspectiva más amplia de la política comercial global de los Estados Unidos.

Ante todo, hay que tener en cuenta que el Acuerdo de Libre Comercio de Canadá y los Estados Unidos tiene un carácter defensivo frente a la Europa del 92. En este sentido, el Acuerdo no es más que un primer paso en la perspectiva de la formación de un bloque continental hegemonizado por los Estados Unidos.

Una primera versión de esta perspectiva la dio el Presidente Reagan cuando en 1985 al anunciar el inicio de las negociaciones con Canadá. El Sr. Reagan dijo entonces que el acuerdo a que se llegara serla el primer paso hacia la formación de un bloque comercial que comprenderia, según sus palabras, "desde el Yukón hasta Yucatán". Cinco anos más tarde el Presidente Bush anunció la "Iniciativa de las Américas", con lo cual se pasó a la definición hemistérica de la respuesta de Estados Unidos al desafio europeo.

Antes de pasar al examen de la mencionada Iniciativa es conveniente tener en cuenta algunos hechos en los que debe encuadrarse al discurso del Presidente Bush.

Ante todo está el hecho de que la política de comercio exterior de los Estados Unidos responde a la pérdida de competitividad y de liderazgo tecnológico frente a Europa y el Japón. Debido a lo anterior los Estados Unidos sufren enormes pérdidas en su balanza comercial, no obstante que ese pais sigue siendo el más grande exportador del mundo. 
Por otra parte, Estados Unidos enfrenta hoy dia el formidable desafio de la Europa del 92 y del proceso de formación de un bloque económico en el Extremo oriente, hegemonizado por el Japón, al que pertenecerán algunos de los más importantes exportadores mundiales, como son la República de Corea, Taiwán, Singapur y Hong Kong.

Ante tales tendencias y desafios la Administración norteamericana otorga creciente protección a los productores domésticos frente a la competencia extranjera y refuerza los mecanismos de represalia para el caso en que, a su juicio, se incurra en prácticas comerciales "no razonables, injustas y discriminatorias".

Ya en la Ley de Comercio de 1974 habia tomado cuerpo esta politica, pero la Ley Omnibus de Comercio y Competitividad, en vigor desde 1988, llevó las cosas mucho más lejos. La nueva ley extendió el ámbito de la negociación a las llamadas "nuevas áreas" (servicios, inversión extranjera y propiedad intelectual), asi como a las políticas de compra gubernamentales y a la violación de derechos laborales.

Sobre ninguna de estas materias hay un cuerpo de normas internacionalmente aceptadas, lo que da origen a frecuentes conflictos tanto con los paises industriales como con los de América Latina. La diferencia entre unos y otros es, obviamente, la fragilidad de la capacidad de contraataque latinoamericano.

Se puede anticipar, casi con completa certeza, que los temas indicados reaparecerán en los acuerdos que negocien los Estados Unidos con paises de América Latina, sobre todo en las negociaciones con Argentina, Brasil y México, con los cuales ha habido periodicamente fuertes tensiones debido a la reserva de mercado para algunas industrias, a la legislación sobre inversiones extranjeras y sobre patentes, y otros asuntos más. Es necesario tener presente to anterior, pues el temor a las represalias de los Estados Unidos, el temor al cierre del mercado norteamericano está detrás del interés que ha despertado en América Latina la posibilidad de llegar a acuerdos de libre comercio con los Estados Unidos. Se busca estabilidad en las pautas de relacionamiento. Esa misma aspiración estuvo presente en la negociación del Acuerdo Canadá-Estados Unidos. En este caso, no obstante que una buena parte de las exportaciones canadienses tenia libre acceso al mercado de los Estados Unidos, en todo momento el gobierno y las empresas canadienses temieron que Washington cediera ante las presiones de los industriales norteamericanos $\theta$ impusiera restricciones al intercambio.

Un rasgo esencial de la posición comercial de los Estados Unidos 
es su elevado proteccionismo. A este respecto se ha hecho notar que una de las razones del Presidente Reagan para apoyar la iniciativa de libre comercio con Canadá, fue que "la formación de un bloque entre los dos mayores socios comerciales del planeta (Canadá y Estados Unidos) otorgaria credibilidad a la política externa de un gobierno caracterizado por la contradicción entre el discurso radical en favor del liberalismo y la práctica del más desmesurado proteccionismo observado en ese país en los últimos 40 años". ${ }^{10}$

Sobre este particular las perspectivas no son más alentadoras. Hay demasiados desacuerdos entre los grandes centros de poder mundial, por lo que no se puede anticipar un abatimiento del proteccionismo en los próximos años. Antes bien, si la Ronda Unuguay del GATT fracasara, que es lo más probable, el Presidente Bush anticipó ya, en su discurso ante la reunión anual del Fondo Monetario Internacional y el Banco Mundial, que el mundo presenciará una nueva escalada de proteccionismo". Es evidente que también en este caso la firma de un acuerdo de libre comercio tiene el atractivo de precaver a la América Latina de la aplicación de las medidas proteccionistas norteamericanas.

Por último, como trasfondo de la Iniciativa de las Américas está el hecho siguiente. No obstante que en términos relativos el comercio de Estados Unidos con América Latina ha tendido a perder importancia, pues ha ganado terreno el comercio con otras regiones, la crisis de los 80 puso de relieve la profunda interdependencia que existe entre Estados Unidos y América Latina. Esto, que es obvio desde la perspectiva latinoamericana, lo es también ahora para la parte norteamericana.

En efecto, es un hecho que la crisis de la deuda externa latinoamericana, al obligar a los paises de la región a reducir drásticamente sus importaciones, ha tenido un efecto demoledor sobre la economia de los Estados Unidos. Se estima que este pais ha perdido entre 50,000 y 130,000 millones de dólares por concepto de la caida de las exportaciones norteamericanas a la América Latina. Ello ha dado por resultado la pérdida de más de un millón de empleos en los Estados Unidos.

Por lo anterior, y ante el desafio de los bloques de Europa y del Extremo Oriente, para los Estados Unidos adquiere una importancia estratégica dominar el mercado latinoamericano. No es extraño, en

10. José Tavares de Araujo Jr., "Integración Económica en América del Norte y en el Cono Sur", Comercio Exterior, 8, México, 1990, p. 740.

11. Excelsior, México, 26 de septiembre 1990, p. 1 y 26, Sección A. 
consecuencia, que el senor William Rodgers, antiguo Secretario del Departamento de Estado y actual Vicepresidente de Kissinger Associates, manifestara ante el Comité Económico Conjunto del Congreso norteamericano: "América Latina es ahora (...) más importante que durante la Guerra Frla". ${ }^{12}$

\section{La Inlciatlva de las Amérlcas}

En el cuadro de lo expuesto anteriormente se inserta la Iniciativa de las Américas. No es mucho lo que se sabe acerca de esta Iniciativa. Se cuenta hasta ahora solo con el discurso del Presidente Bush el 27 de junio de 1990 y con las declaraciones preliminares de algunos funcionarios norteamericanos.

Se sabe que la Iniciativa fue preparada con gran confidencialidad por un grupo reducido de funcionarios; no hubo reuniones interagenciales de la administración norteamericana para elaborar la nueva política; por tanto, no se han precisado muchos aspectos del plan presidenial sino hasta ahora se comienza a trabajar en éstos en las Oficina del Representante Comercial de los Estados Unidos. Debido a to anterior tampoco se cuenta todavia con propuesta al Congreso de la Unión.

Sin embargo, el hecho de que haya sido el propio Presidente Bush el que hizo la declaración, y no uno de sus ministros, le confiere a la Iniciativa un gran respaldo político. De inmediato la Oficina del Representante Comercial se puso a trabajar, se concluyeron acuerdosmarco con Bolivia, Colombia y Ecuador para sentar las bases de futuras negociaciones, y se está trabajando en los diversos aspectos que se habrán de considerar en los acuerdos de libre comercio.

La iniciativa fue presentada en los siguientes términos por el Presidente Bush: "...Los tres pilares de nuestra Iniciativa son el comercio, la inversión y la deuda. Para expandir el comercio propongo que demos comienzo a un proceso de creación de una zona de libre $\infty$ mercio que abarque a todo el hemisferio. Con el proposito de incrementar la inversión, que adoptemos medidas encaminadas a promover el flujo de nuevo capital hacia la región, y para aliviar aún más la carga de la deuda, que adoptemos un nuevo enfoque con respecto al endeudamiento regional, con beneficios importantes para el medio ambiente".

No podemos en esta oportunidad extendernos al análisis de los tres pilares de la Iniciativa. El asunto es por si solo materia para otra disertación. Muy brevemente podria decirse lo siguiente:

12. Citado en: La Iniciativa Bush para las Américas: Análisis Preliminar de la Secretarla Permanente del SELA, 1990, p. 15. 
El elemento trascendental de la propuesta es el relativo a la zona hemisférica de libre comercio. Ella sola ha abierto un nuevo capltulo en la historia de las relaciones con los Estados Unidos, en el curso de la cual, de igual manera que en el pasado, junto a las coincldenclas habrán de manifestarse muchas contradicciones. Los otros dos pllares (los relativos a la inversión y la deuda) resultan de una pequenez mezquina al comparárseles con los problemas a los que hacen referencia.

El tema de las inversiones para una América Latina descapitalizada es como hablar de forraje donde hay ganado flaco. Pero la oferta es extremadamente modesta. Se propone abrir una nueva linea de credixo para inversiones en el Banco Interamericano de Desarrollo, que tendrla por objeto proveer asesorla técnica y respaldo financiero para la privatización de empresas públicas. Asimismo, se proyecta crear un fondo de inversiones, por el plazo de cinco anos, al cual contribuirán los Estados Unidos con la suma de 100 millones de dollares anualmete, Japón aportarla otros 100 millones, y una suma igual la comportirlan de alguna manera el Canadá y Europa. O sea, un aporte anual total de 300 millones de dolares, que es equivalente a lo que América Latina ha pagado en el perlodo 1982/89 por servicio de la deuda externa en solo 4 dlas.

Pese a su modesta magnitud la constitución de este fondo es todavla muy incierta. En primer lugar, el Presidente Bush todavla no ha solicitado los fondos al Congreso norteamericano. En segundo lugar, cuando el Presidente Bush presentó la propuesta en la Reunión Cumbre realizada en julio pasado en Houston (Estados Unidos), ninguno de los Jefes de Estado participantes quiso asumir compromiso abuno. Esta fria renuencia contrasta con la celeridad con que los mismos palses participantes en la Reunión Cumbre, junto a otros palses Industriales, decidieron aportar 12,000 millones de dolares para crear el Banco Europeo para la Construcción y el Desarrollo, destinado a proveer financiamiento a la Unión Soviética y los palses de Europa Oriental.

Por lo que toca al pilar de la reducción de la deuda podrla decirse que toda iniciativa para reucir su carga debe ser recibida con Interés pero también en esta materia la propuesta es bastante discreta. No se aborda el tema de la deuda privada, sino solamente el de la deuda contralda por América Latina con el gobiemo norteamericano, que asciende a un total de 12,000 millones de dólares. O sea, apenas el $3 \%$ de la deuda extema total de América Latina, que asciende a la 
fecha a 417,000 millones de dólares, de acuerdo a las estimaciones del Banco Mundial.

Cualquiera que sea el alcance de las propuestas sobre inversión y deuda extema debe acogerlas América Latina con interés, en forma estrictamente pragmática, persiguiendo el objetivo de que se amplien en 10 posible y se adecuen de mejor manera a los intereses latinoamericanos. Sin embargo, lo que debe concentrar la atención latinoamericana es el elemento comercial de la Iniciativa de las Américas.

Hasta ahora la política de los Estados Unidos hacia América Latina ha estado dictada por razones de seguridad. Desde la terminación de la II Guerra Mundial hasta el ano pasado el tema dominante fue la confrontación de los Estados Unidos y la Unión Soviética. Esto hoy dia ya es tema para historiadores. Ahora son razones económicas (la carrera por el liderazgo comercial y tecnológico mundial con Europa y el Japón) las que han inspirado el cambio más trascendental que podia esperarse de la política comercial norteamericana hacia América Latina.

Al subrayarlo nos anima el propósito de subrayar igualmente la elevada responsabilidad de los gobiernos latinoamericanos de prepararse a fondo para las negociaciones con los Estados Unidos. Hasta ahora ha habido mucha inquietud por interpretar lo que quiso decir el Presidente Bush. Nos parece que esta tarde hay que dejarla a sus asesores. Lo que América Latina tiene que hacer es elaborar su propia estrategia de negociación, precisando con el más sólido fundamento los intereses que tendrá que defender en las negociaciones.

Sobre este particular hay por ahora muchas interrogantes. El escenario está planteado. Comienzan a moverse los actores. Algunos países ya han firmado o están para suscribir acuerdos-marco con los Estados Unidos, los cuales establecen el procedimiento para abordar el proceso de negociación, pero sin que ninguno incluya concesiones en materias sustantivas.

Es muy prematuro anticipar la secuencia que seguirán las negociaciones en torno a la integración hemisférica; ni cuanto tiempo tomará concretarla; ni la forma que asumirá; ni la extensión de las concesiones recíprocas; ni las reservas que ambas partes hagan de aquellas áreas que consideren estratégicas para sus intereses nacionales. Lo que si puede anticiparse es que a todos los países les favorecería negociar a partir de una reflexión regional sobre la problemática. Partiendo del reconocimiento de la diversidad de intereses y de la disimilitud de las relaciones comerciales de cada país con los Estados Unidos, debe ser posible armonizar algunas orientaciones, pues para todos es común la 
premisa de que en una hipotética integredon con el bloque del norto América Latina ocupa una posición defenstva ante podores my superiores a su dimensión económica.

La integración regional es una respuesta al desalfo de la integrecton hemistérica. Hay tiempo para actuar. Serla ingenuo pensar que la zona de tore comercio se concretará en un corto plazo. El propio Presidente Bush hable de plazos langos. Cuan largos sean no dependerd slquiera de la administración norteamericana, pues falta aun que se manifiesten plenamente las controversiales posiciones del Congreso y de los sindicatos y de las empresas norteamericanas. $Y$ falta asimismo que se definan las posiciones de América Latina. Cuando ya se esté pisando terreno concreto seguramente emergerán muchos de los mottvos de desacuerdo polltico y económico que históricamente nos han distanciado de los Estados Unidos.

Durante el plazo más o menos prolongado que medie entre el presente y la integración que se anticipa, la integración regional constituye tanto una respuesta a los desaffos del mundo industrial como una estrategia que responde al imperativo de crear, ampliar y consolidar una capacidad autónoma de crecimiento.

Desde su formulación en los anos $\mathbf{5 0}$ la teorla latinoamericana de integración ha puesto el énfasis en el desarrollo mediante los recursos de la región, pero este volverse de América Latina hacia si misma no ha sido nunca una alternativa a su extroversión hacia el resto del mundo.

Hoy dia ha adquirido una nueva dimensión, mucho mayor que en el pasado, el relacionamiento con el resto del mundo, pero ello no subestima los viejos objetivos. Asi se pone particularmente de manifiesto ante el reto fundamental que nos plantea el mundo industrializado: el reto de la productividad y la competitividad; el reto de la tecnologla. En esta materia la integración latinoamericana tiene una nueva oportunidad de evidenciar sus ventajas. Permítasenos dar término a esta exposición con to que digamos sobre este particular.

\section{Integración y desarrollo tecnológlco}

El punto de partida es la constatación harto conocida de que América Latina es una región exportadora de bienes primarios, cuyo comercio internacional tiene las más bajas tasas de crecimiento. Algunos de los productos de exportación latinoamericana más importantes, $\infty$ mo son el petróleo y sus derivados, los cereales, las materias primas agricolas de uso industrial, el azúcar y otros más, se encuentran en la 
lista de los diez productos que pierden rápidamente importancia en ol comercio Intemacional' ${ }^{\prime 3}$.

En el otro extremo se sitúan los productos que tienen una tendencia sumamente dinámica en el comercio mundial, para producir los cuales América Latina deberá incorporar tecnologlas modernas, de diseno muy avanzado por lo general.

Las condiciones para adquirir esas tecnologlas se han vuelto cada vez más restrictivas. Los gobiemos de los principales paises industriales están poniendo en práctica una forma muy agresiva de lo que se ha dado en llamar "neomercantilismo de alta tecnologla". La anterior hegemonia tecnológica de los Estados Unidos viene siendo desplazada por "un oligopolio mundial, heterogéneo y attamente inestable, en el cual las empresas norteamericanas, japonesas y europeas lucharán entre si por colocarse a la cabeza". ${ }^{14}$

La mayor parte de los paises de América Latina no tienen capacidad instalada para penetrar en este mercado agresivamente oligopolizado, a menos que potencien sus capacidades nacionales mediante acuerdos de integración regional. Lo anterior no tiene en cuenta solo a los palses pequenos y medianos, sino es también cierto, particularmente para productos de alta tecnologla, para los paises latinoamericanos de mayor grado de desarrollo relativo (Argentina, Brasil y México).

Si desarrollan sus recursos humanos, si amplian la infraestructura técnica y cientifica y acumulan los medios financieros indispensables, en todo lo cual puede dar una gran contribución la integración regional, los paises latinoamericanos tienen posibilidades de acceder al oligopolio mundial de tecnologla en diversas ramas industriales. Aparte de tecnologlas avanzadas en la industria del vestuario, de la textil de tejidos planos y otras más en que el cambio tecnológico ha sido muy acelerado, el espectro de posibilidades incluyen "la construcción naval, el acero, los vehículos y sus componentes, los productos electrónicos de consumo (incluyendo los grabadores de cintas de video), los periféricos para computadores y (aunque con algunas interrogantes) los microcomputadores y los materiales para semiconductores (especialmente las denominadas D-RAMs, memorias de disco de acceso alea-

13. Ver, Osvaldo Rosales, "Competitividad, productividad e inserción externa de América Latina", Comercio Exterior, 8, México, 1990, cuadro 10, p. 715.

14. Dieter Ernst, Tecnologia y Competencia Global: El Desaffo Futuro para las Economlas de Reciente Industrialización", Pensamiento Iberoamericano, 16, Madrid, 1990, p. 20. 
torio), por mencionar solo algunas de la larga lista de posibilidados". 16

Asimismo, sl la integración regional se concreta en acuerdos para sumar recursos y reconocimientos, Anérica Latina podrá alcanzar ntveles de excelencia en ingenierla de manufacturas, "...inchuso en áreas allamente sofisticadas como la ingenierla de "software" y el diseno de circuitos (...) (si se llega) a acumular suticiente capacidad tecnolbgica on, digamos, los proximos cinco anos". ${ }^{10}$

No obstante que en materia tecnologica las perspectivas son bastante sombrlas, cabe la posibilidad de que el acceso al oligopolio norteamericanos japonés-europeo se facilite, debido a que el crecimiento de esas economlas depende en forma creciente de la expansión de la demanda mundial a sus industrias de alta tecnología y los servicios conexos. Si esa demanda decayera, las consecuencias de esta pérdida de mercados serlan muy graves para los palses industriales. Es de esperar que asl lo comprendan éstos y que, en consecuencia, los palses latinoamericanos, individualmente 0 como conjuntos integrados, tengan la posibilidad de adquirir tecnologla avanzada o de tener acceso a las mismas por la vía de la importación de bienes de capital o de la inversión extranjera directa, a condición de que ésta no impida el acceso a la tecnología que incorpora, como ahora suele ocurrir en muchos casos.

\section{Reflexiones finales}

Me he extendido quizás demasiado y aun asi solo he podido comunicarles algunas ideas, en forma obviamente imperfecta. Debernos admitir que el pensamiento progresista hasta ahora comienza a transitar una etapa de reelaboración teórica y que la dimensión de los cambios en curso han superado toda imaginación. La teorla de la integración latinoamericana, y ahora la innovación introducida por la Iniciativa de las Américas, reclaman de nosotros una cuidadosa revisión conceptual, operativa y estratégica.

Hay algo, sin embargo, que no parece haber sido cuestionado en los hechos, y es que la integración latinoamericana, bajo nuevas formas y con nuevos objetivos, debe estar al servicio de los intereses propios de la América Latina. Una integración para contraponerse al mundo seria una pretensión ridícula, pero tampoco se puede incurrir en la ingenua falacia de la identidad de intereses con el mundo superdesarrollado. El centro remodela el mundo a imagen de sus intereses. La remodelación de la periferia le es impuesta. Desde este punto de

15. Bid., p. ?1

16. Idem. 
vista, la integracion latinoamericana ha sido y sigue siendo defenstva. De los propís latinoamericanos dependerá conquistar una posición decorosa; primeros entre iguales donde sea posible; pero en todo caso que la subaltemidad no sea ni gratuita ni humillante.

México, D.F., a 8 de noviembre de 1990. 